\title{
Using Eye-Tracking to Support Interaction with Layered 3D Interfaces on Stereoscopic Displays
}

\author{
Florian Alt ${ }^{1,2}$, Stefan Schneegass ${ }^{2}$, Jonas Auda $^{2}$, Rufat Rzayev $^{2}$, Nora Broy ${ }^{1,2}$ \\ ${ }^{1}$ University of Munich \\ Media Informatics Group \\ Amalienstraße 17, 80333 München \\ \{firstname.lastname\}@ifi.lmu.de \\ ${ }^{2}$ University of Stuttgart \\ Institute for Visualization and Interactive Systems \\ Pfaffenwaldring 5a, 70569 Stuttgart \\ \{firstname.lastname\}@vis.uni-stuttgart.de
}

\begin{abstract}
In this paper, we investigate the concept of gaze-based interaction with 3D user interfaces. We currently see stereo vision displays becoming ubiquitous, particularly as autostereoscopy enables the perception of 3D content without the use of glasses. As a result, application areas for 3D beyond entertainment in cinema or at home emerge, including work settings, mobile phones, public displays, and cars. At the same time, eye tracking is hitting the consumer market with low-cost devices. We envision eye trackers in the future to be integrated with consumer devices (laptops, mobile phones, displays), hence allowing the user's gaze to be analyzed and used as input for interactive applications. A particular challenge when applying this concept to 3D displays is that current eye trackers provide the gaze point in 2D only ( $\mathrm{x}$ and y coordinates). In this paper, we compare the performance of two methods that use the eye's physiology for calculating the gaze point in 3D space, hence enabling gaze-based interaction with stereoscopic content. Furthermore, we provide a comparison of gaze interaction in 2D and 3D with regard to user experience and performance. Our results show that with current technology, eye tracking on stereoscopic displays is possible with similar performance as on standard 2D screens.
\end{abstract}

\section{Author Keywords}

Eye tracking; 3D; gaze interaction; stereoscopic displays

\section{ACM Classification Keywords}

H.5.2 Information Interfaces and Presentation: User Interfaces-Input devices and strategies

\section{INTRODUCTION}

Today, 3D technology is widely used for displays in an entertainment context, for example to play games or to watch movies in cinema or at home. At the same time, 3D is used in specialized work environments, where 3-dimensional

Permission to make digital or hard copies of all or part of this work for personal or classroom use is granted without fee provided that copies are not made or distributed for profit or commercial advantage and that copies bear this notice and the full citation on the first page. Copyrights for components of this work owned by others than the author(s) must be honored. Abstracting with credit is permitted. To copy otherwise, or republish, to post on servers or to redistribute to lists, requires prior specific permission and/or a fee. Request permissions from Permissions@ acm.org.

IUI'14, February 21-27, 2014, Haifa, Israel.

Copyright is held by the owner/author(s). Publication rights licensed to ACM ACM 978-1-4503-2184-6/14/02?\$15.00.

http://dx.doi.org/10.1145/2557500.2557518

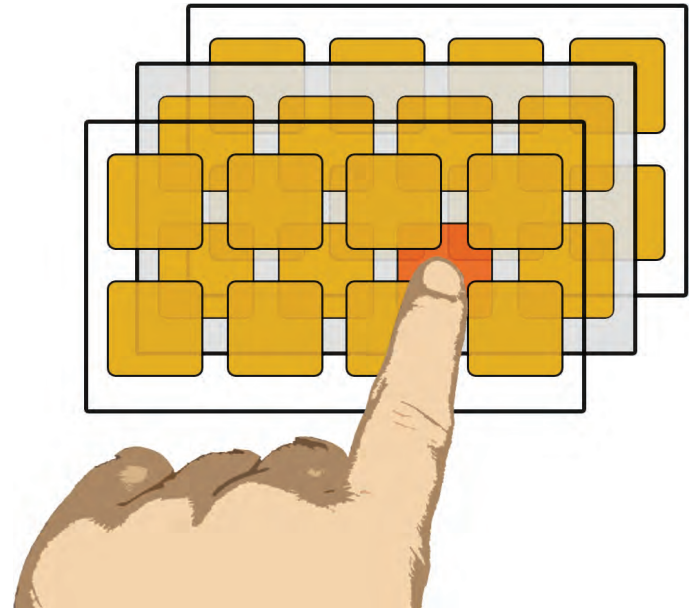

Figure 1. By using gaze to select the specific layer (gray), the user is able to interact with this layer in the same way as with $2 D$ interfaces.

data needs to be analyzed, including CAD and medical work places. A major reason that hampers the widespread usage in other domains is the need for glasses in cases where anaglyph, polar, or shutter technology is used to create the 3D effect.

More recently, auto-stereoscopic displays entered the consumer market, making it possible to perceive content in 3D without the use of glasses. First examples, where this technology is already integrated into consumer devices include mobile phones and public displays. In such interfaces, depth can be used to highlight important information or to group information that belongs together by means of its position along the $\mathrm{z}$ axis. Such interfaces, that aim to enhance the users' focused attention by arranging them on distinct planes are commonly referred to as layered user interfaces [10].

At the same time, devices to track the users' gaze are becoming commercially available. Leading manufacturers recently released low-cost eye trackers, making it likely that such devices will be soon integrated with devices like laptops or displays. These devices do not only make it possible to passively monitor a user's gaze behavior but enable gaze-based interaction. For example, an interface could be adapted based on attention or users could explicitly control an application. 
Using standard input devices, like touch screens, for interacting with 3D user interfaces is cumbersome due to the lack of a third (physical) dimension. Hence, we see particular potential in using gaze as an (additional) input modality. In this way, for example, a specific layer could be selected using gaze. Hence, the interaction space is reduced to the wellunderstood 2D space. The major challenge of this approach is to apply eye tracking to 3D, as state-of-the-art eye-trackers usually provide the gaze point in 2D space only. In this work, we compare two approaches based on the physiology of the pupil to detect the correct depth position in 3D space.

The contribution of this paper is threefold. First, we introduce the concept of interaction with layered 3D user interfaces, sketching particular application domains that could benefit from gaze as an input modality. Second, based on a study with 14 participants, we provide a comparison of two methods to calculate the gaze point in 3D space with regard to user performance. Third, we compare the user experience and performance of gaze interaction in 2D to gaze interaction in 3D.

\section{RELATED WORK}

With the advent of 3D displays, researchers started to investigate how user interfaces could benefit from this technology. Sunnari et al. investigated the user experience of 3D menus on mobile phones with auto-stereoscopic screens compared to traditional 2D displays [20]. They found that users perceived 3D menus to be more visually pleasing, entertaining, innovative, and empowering but also to be more time-consuming and difficult to control. McIntire et al. investigated human performance on stereoscopic 3D displays [17]. They found such displays to be particularly beneficial for depth-related tasks, such as judging distances or finding and identifying objects. Furthermore, stereoscopic displays improve recall of scenes and objects as well as learning of spatial relationships. On the downside, 3D visualizations were found to cause eyestrain, headache, and fatigue if not carefully applied. To avoid this, Broy et al. showed how to determine a comfort zone for layered UIs and provide insights into how to structure the displayed information in different layers in a way that increases comprehensibility [5]. Huhtala et al. showed that layering information can be used to reduce search times for information [13]. In summary, these findings show a strong benefit of 3D for accessing information if applied carefully.

Whereas 3D information is nowadays often displayed statically, researchers also looked into novel techniques to interact with the content. Direct interaction with 3D content has been investigated within the Holodesk project [12]. The Holodesk is based on a 3D projection from the top and uses depth sensors (i.e., Microsoft Kinect) to track the users' hands. Interaction with volumetric 3D displays using touch gestures has been introduced in [8]. In both cases, interaction is strongly tailored to the device. The reason is that techniques known from 2D user interfaces are in general difficult to apply to 3D. As eye trackers become commercially available, we believe that gaze as a complementary input technique could be one possible solution to this challenge. With gaze users could select a particular depth layer and then interact with it by using techniques commonly applied in 2D user interfaces.
Gaze interaction has been subject to research in several domains. Kern et al. showed how to use gaze for implicit and explicit interaction in the car [15]. Recently, eye tracking in front of public displays has received considerable attention. So far, research mainly focussed on implicitly calibrating eye trackers for passersby that approach a display [21]. Castellina et al. applied eye tracking to virtual 3D games and suggested different multimodal gaze interaction techniques. However, all those applications apply eye tracking on 2D displays only.

Research on gaze interaction with stereoscopic displays, and hence with real 3D content, is rather scarce. Prior work has looked at enabling techniques to assess gaze on stereoscopic displays. This work shows that tracking the gaze point in 3D space is in general possible by using the physiology of the eye, particularly the distance between the pupils $[2,7]$ and the pupil diameter [19]. Application of gaze tracking on stereoscopic displays includes the work of Ramasamy et al. who looked into how stereoscopic movies could be analyzed using gaze information [18]. Interaction using gaze for $3 \mathrm{D}$ displays has been explored by Ki and Kwon in [16] who control a dart game on an auto-stereoscopic display with the user's gaze.

In summary, the review of related literature shows a potential for the use of 3D technology for layered user interfaces. At the same time, eye tracking as an emerging technology is promising as a (complementary) interaction modality for such interfaces. Eye tracking in 3D has so far only been applied to a few application areas - both for analysis and interaction. Particularly for interaction, data on user performance is scarce and has as of today only been assessed qualitatively [16]. We are neither aware of any work that compared user performance and subjective perception of 2D gaze interaction to 3D gaze interaction, nor of work that compared the performance of different gaze point calculation methods. In the remainder of this paper we present our design of a prototype for gaze-based interaction on a stereoscopic display and a quantitative evaluation comparing $2 \mathrm{D}$ to $3 \mathrm{D}$ gaze interaction as well as different gaze point calculation methods.

\section{SUPPORTING LAYERED 3D INTERFACES}

Prior work has shown a combination of salient features (e.g., color and depth) to significantly decrease search times $[1,13]$. A UI can hence employ depth layers to group or highlight urgent or frequently used objects. To then interact with objects on one layer we envision a multi-modal approach in which gaze is used to select a specific layer and then apply commonly used input methods (e.g., touch or keyboard and mouse) to perform actions within the selected layer (cf., Figure 1). Note, that by applying this approach, interaction is in general also possible using gaze only, e.g., by looking at an object for a certain amount of time and, thus, executing an action. In the following, we present four use cases in which our concept can be applied to ease interaction with 3D UIs.

\section{In-car Displays}

In-car interfaces have specific requirements in terms of how the user operates them [6]. While the primary goal is to keep the driver's attention on the road, the dashboard provides different types of information, necessary to operate the vehicle. 
These include warnings (fuel status, doors open, handbrake not released), navigation cues, status information (temperature, time), traffic information or the currently tuned in radio station. Introducing 3D displays can decrease the cognitive load by presenting information on different layers in an easy and quick-to-perceive way. Gaze could simplify interaction through fast and intuitive interaction techniques (e.g., selection of a layer and object via gaze and execution via a button on the steering wheel).

\section{Displays in Work Environments}

Specialized work environments already employ stereoscopic displays. For example, in medical environments, 3-dimensional information in the form of layered MRT images (magnetic resonance tomography) is frequently used. During surgeries, medical staff often needs to navigate through the data but cannot do so using touch for hygienic reasons [14]. In these cases, gaze could be used to easily navigate through and access information on particular layers.

\section{Public Displays}

Popular interaction techniques for public displays include touch, gestures, and smartphones. However, many users have reservations against using touch in public due to a lack of sanitation after each user. Gestures are often cumbersome to be used for selection due to low accuracy. And smartphones are slow as they need to be taken out of the pocket and many users are not willing to install software. As a result, gaze as input modality for public displays has recently received considerable attention [21]. At the same time, the advertising industry started to deploy auto-stereoscopic displays at airports and in train stations ${ }^{1}$. We envision this technology to be soon deployed also for information displays, where users could access transport schedules, store directories, or city maps. Information could be presented on different layers and as displays employ sensors, users could control them via gaze.

\section{Mobile Interfaces}

Mobile devices have small displays and, thus, information placement is challenging. By integrating $3 \mathrm{D}$ displays the third dimension can be used to group the information on different layers (e.g., contact lists [9] on one layer and call logs on another layer). Still, small displays and touch interaction make selection cumbersome. We believe gaze to simplify interaction as it enables quick and accurate selection while at the same time allowing standard controls to be used.

\section{APPARATUS}

To enable 3D gaze interaction on stereoscopic displays and compare it to $2 \mathrm{D}$, we implemented a prototype that allows gaze information to be obtained from an eye tracker, calculate the gaze position in 3D space using different methods (pupil diameter and pupil distance), and make it available to client applications. The following section introduces the architecture, describes the used gaze point calculation methods, and presents a sample application.

\footnotetext{
${ }^{1}$ Real Eyes Website: http://real-eyes.eu/
}

\section{Architecture}

Our prototype consists of an eye tracker, a gaze server that collects and processes gaze data, and a client application. The SMI RED eye tracker samples gaze data at $60 \mathrm{~Hz}$. An API allows the server to obtain different types of data and process it for the use in a client application. Types of data include the gaze position on the screen (x/y coordinate) for each eye, the gaze direction, and the diameter of each pupil. The communication between the components is based on UDP. The gaze server takes the gaze data, calculates additional values, such as the ocular vergence and sends them to the client. 3D client applications are created in $\mathrm{Unity}^{2}$ using C\#. The gaze point calculation is done within the client application. We use Unity $3 \mathrm{D}$ rays to determine the intersection of the user's gaze with an object, allowing layers or elements to be selected.

\section{Calculating Gaze Points in 3D Space}

For 2D, eye trackers calculate the $\mathrm{x}$ and $\mathrm{y}$ coordinate of the user's gaze point on the screen. In contrast, the depth (expressed as z-value) is needed to obtain a gaze point in 3D space. Different approaches have been reported to calculate this gaze point. In simple cases, where only one object is in the line of sight, an application could simply calculate the intersection of the gaze direction with the object. However, when multiple (semi-transparent) objects appear in the line of sight, more fine-grained methods are required. In this work, we implemented two such approaches.

Pupil Diameter The pupil diameter technique exploits the fact that, based on the distance of an object that is in focus, the diameter of the pupil changes, given that lighting conditions remain constant [19]. The pupil diameter ranges from 2 to $6 \mathrm{~mm}$ under normal photopic conditions $\left(>3 \mathrm{~cd} / \mathrm{m}^{2}\right)$, where the largest depth of field is obtained at $2 \mathrm{~mm}$. Hence, by measuring the pupil diameter a system can calculate the depth position (or depth layer) on which the user focuses.

Pupil Distance An alternative method considers ocular vergence, that is the distance between the left and right eye pupil caused by the simultaneous inward rotation for the eyes towards each other $[2,7]$. As the user focuses on an object closer to the eye, the distance decreases compared to situation where the user focuses on an object that is located further away. Prior work found this method to work best for objects near screen level [7].

The strength of the pupil diameter method is that it is applicable both to monocular as well as to binocular eye trackers, whereas the pupil distance requires a binocular device.

\section{D Calibration}

As the cornea of each user differs, eye trackers need to be calibrated. Standard procedures depict a number of points on the screen the user needs to focus on, e.g., a regular $3 \times 3$ grid. We extend this procedure to the z-dimension by depicting calibration points also on two different depth layers. This results in $3 \times 3 \times 2$ equally distributed calibration points. In addition we store the pupil diameter and the pupil distance for each calibration point.

\footnotetext{
${ }^{2}$ Unity Website: http://unity3d.com
} 

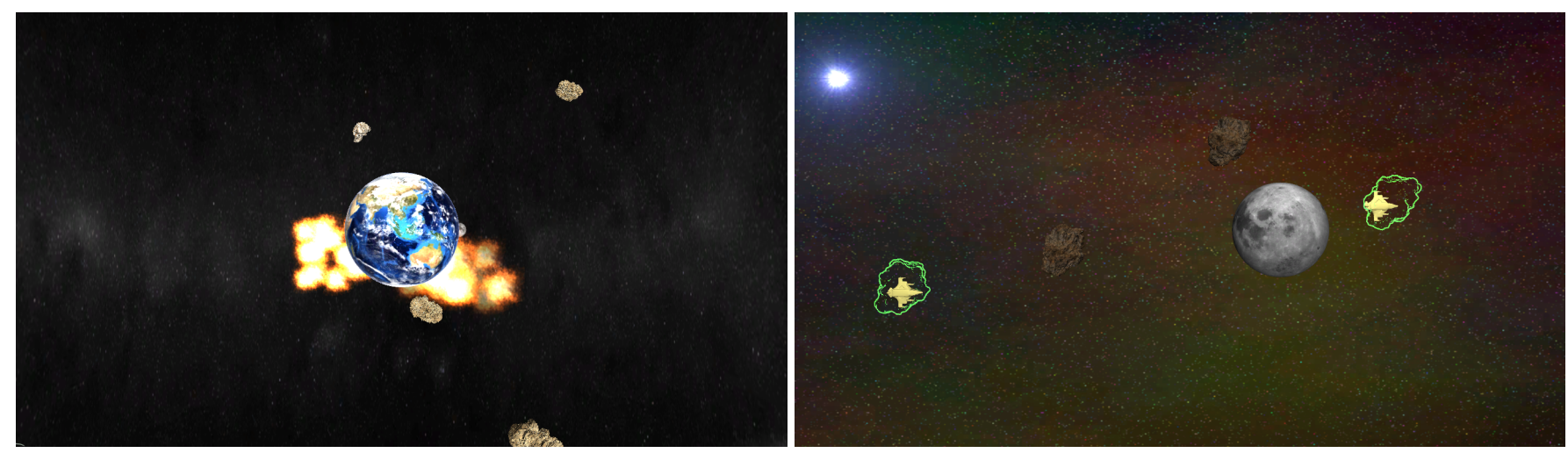

Figure 2. In the first level (left), meteoroids are flying towards earth from different angles. In the second level (right), enemy space ships are flying from left to right hiding behind meteoroids on a different depth layer.

\section{Sample Application: Earth Defender}

For our anticipated user study we implemented a simple game where users can interact with objects on different depth layers using gaze. We deliberately decided to design a playful application instead of a simple pointing task as we believe users to behave more natural when playing the game. In this way, we aim to increase the ecologic validity of our evaluation while maintaining a controlled setting.

Earth Defender is a space shooter where players need to destroy meteoroids and enemy space ships trying to attack earth. We implemented two different levels. In the first level - the meteoroid level - a number of meteoroids (cf. Figure 2, left) that simultaneously try to hit earth need to be destroyed via gaze (i.e., by looking at the meteoroids). The number of meteoroids, and hence the difficulty, increase throughout the levels. In the first step, only a single meteoroid approaches earth, in the second step two meteoroids, and so on. The game is over when ten meteoroids managed to hit earth. In the second level - the enemy space ship level - evil space ships hide behind meteoroids and need to be destroyed by the player using gaze before leaving the viewport (cf. Figure 2, right). Enemy space ships and meteoroids are placed on different depth layers. As enemy space ships hide behind meteoroids, the latter become semi-transparent so that the meteoroids and the space ship can be seen by the player. In cases where the player destroys the meteoroid instead of the hiding space ship, the space ship accelerates quickly, making it difficult to aim at it. Similar to the first level, the number of enemy space ships increases as players successfully destroy them. The game ends as ten space ships escaped the viewport.

Note that the first level can be played both in 2D and 3D, because there are no overlapping elements on the screen. Hence, we simply calculate an intersection of the user's gaze with the meteoroid to detect a hit. The second level can only be played in 3D, because elements are positioned behind each other. Either the pupil diameter method or the pupil distance method can be used to calculate the gaze point and detect a hit.

\section{EVALUATION}

The aim of our user study was twofold. First, we wanted to compare performance and user perception of gaze interaction with $2 \mathrm{D}$ user interfaces to interaction with $3 \mathrm{D}$ user inter- faces. Second, we wanted to compare user performance for the two gaze point calculation methods for cases where elements are obscuring each other. We hence used the Earth Defender game which requires users to accurately aim at a target (the enemy space ships and the meteoroids) and to be as quick as possible. As metrics we used the number of destroyed meteoroids or space ships (i.e., the number of correctly selected targets) and the time between the target entered the scene and when it was destroyed. For the comparison between interaction in $2 \mathrm{D}$ and $3 \mathrm{D}$, we additionally assessed usability, task load, and user experience.

\section{Apparatus and Procedure}

For the evaluation, we setup the Earth Defender game on a 55" 3D LCD TV that uses polarization glasses. The stationary SMI RED was placed in front of the user.

In the days prior to the study we recruited participants via mailing lists. As they arrived at the lab, we led them to the room where we had setup the prototype. We briefed them about the study and had them sign a consent form. After that, they filled in a demographic questionnaire on age, gender, and profession. Next, the eye-tracker was calibrated with our 3D calibration procedure. To test the calibration accuracy, nine meteoroids were shown that needed to be destroyed. If the participant was able to destroy all meteoroids, they started playing the game; else the calibration was repeated.

For the first level, participants played in 2D and 3D mode wearing the polarization glasses for both conditions. The conditions were counterbalanced, thus, half of the participants started in 2D mode before proceeding with 3D and vice versa. In each condition, participants played for 3 minutes, before filling in a questionnaire on user experience (Product Reaction Cards; PRC [4]), usability (System Usability Scale; SUS [3]) and task load (Nasa Task Load Index; NasaTLX [11]).

After that, participants proceeded with level two where they played in $3 \mathrm{D}$ using the pupil distance and the pupil diameter method (3 minutes each), again in counter-balanced order. We neither told participants about the two different methods nor provided technical details. After completing both conditions asked participants, which version they preferred. In addition we logged user performance in each condition. 


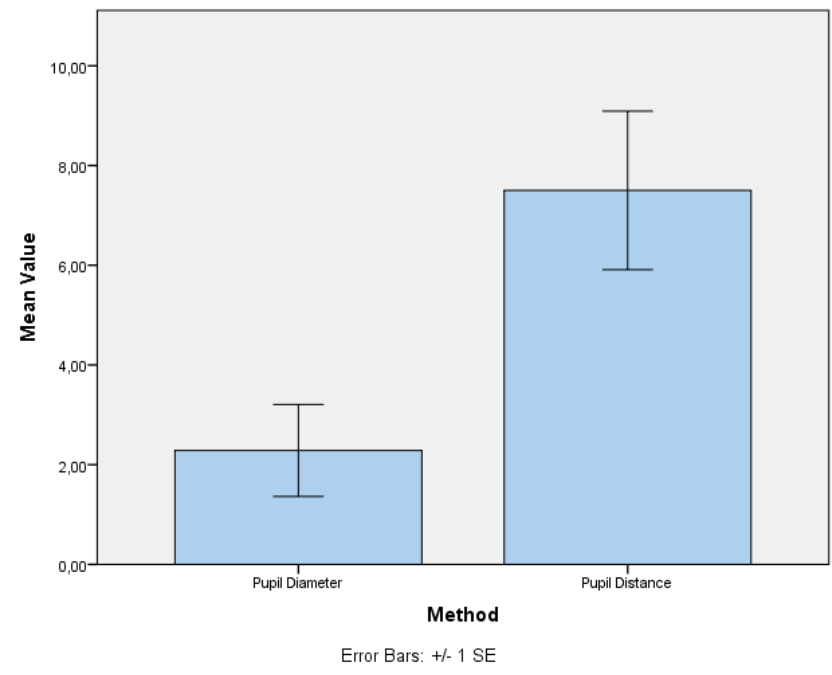

Figure 3. Comparing the number of successfully destroyed meteoroids of the pupil diameter and distance of pupils method.

\section{Results}

In total, 15 users (14 male, 1 female) aged from 22 to 28 years ( $M=24.71, S D=1.73)$ participated in the study. We had to exclude the data from one male participant due to tracking problems. Each participant had normal or corrected to normal eyesight and was able to perceive stereoscopic 3D.

\section{Comparing $2 D$ vs. $3 D$}

In the first part of the study, we compared interaction in 2D versus 3D. Neither the results from the SUS questionnaire used to assess usability $\left(\mathrm{SUS}_{2 D}: 74.53 ; \mathrm{SUS}_{3 D}: 74.33\right)$ nor from the Nasa TLX questionnaire used to assess the cognitive load on the user $\left(\mathrm{NasaTLX}_{2 D}: 54.4\right.$ NasaTLX $\left._{3 D}: 53.3\right)$ showed any statistically significant differences. The results of the PRCs show an effect on the user experience. While the game speed was the same in both conditions, the interaction in 2D was perceived much faster compared to interaction in 3D. Furthermore, participants considered 3D interaction to be more visually pleasing and entertaining.

Finally, we compared the accuracy (number of successfully destroyed meteoroids) for all users. We found that participants performed slightly better in the 2D condition $(M=$ $58.57, S D=17.54)$ than in the $3 \mathrm{D}$ condition $(M=53.79$, $S D=14.83)$. However, based on the results of a $t$ test, statistical significance can not be reasoned, $t(13)=1.01, p=.33$.

\section{Pupil Diameter vs. Pupil Distance}

When comparing the two gaze point calculation methods (cf., Figure 3), we found that participants performed better in the pupil distance condition $(M=7.50, S D=5.95)$ than in the pupil diameter condition $(M=2.29, S D=3.45)$. Due to a non-normal distribution of the results, we performed a nonparametric Wilcoxon tests showing statistically significant differences between the conditions, $Z=-2.138, p=.03$.

Looking closer at the results, the participants' accuracy increases over time using the pupil diameter method. This indicates that some kind of learning is necessary to interact with this technique. Hence, the pupil diameter technique may be better suited for daily use, such as interaction with the mobile phone, than for interactions that happen less frequently, such as interaction with public displays. In addition, we found that the performance of the participants with the pupil distance method varies depending on the position of the space ship.

\section{DISCUSSION}

Our results suggest that in general gaze interaction in $2 \mathrm{D}$ is perceived equally usable and demanding as interaction in 3D. This is also backed by the fact that we did not find any significant difference in accuracy. We hence assume that tasks users currently perform in $2 \mathrm{D}$ could quite easily be performed also in $3 \mathrm{D}$ environments. Future work could look at the effects of multi-modality, for example, how performance is affected in cases where users interact with an interface based on gaze and an additional input modality. Findings from the user experience evaluation suggests that cases where task completion time is crucial could benefit from gaze-based 3D interaction as it is perceived slower by users. With regard to the gaze point calculation method, our results show that simple intersection of the gaze vector with an object works equally well in $2 \mathrm{D}$ and $3 \mathrm{D}$. In cases of overlapping objects that require additional depth information, pupil distance outperforms the pupil diameter. Nevertheless, the accuracy when interacting using the pupil diameter method might increase in the long term, making this approach particularly suitable for cases where only monocular tracking is possible.

\section{LIMITATIONS}

We acknowledge the following limitations of our user study. First, for simplicity we only evaluated two different depth layers, leaving a thorough analysis of the impact of depth and number of layers for future work. Hence, we can not generalize our findings to an arbitrary number of layers and to cases in which objects are shown at extreme parallaxes. Nevertheless we covered a use case suitable for a multitude of applications with a limited number of depth layers at a comfortable viewing position of the user.

Second, we observed an impact of environmental influences. The pupil distance seems to be more robust to slight variances in the lightning conditions or head movement, whereas the pupil diameter seems to suffer from these changes. To obtain comparable results we ran the study under controlled lab conditions (minimal changes in position of the user and lighting). Hence, we cannot claim that the results of our evaluation generalize, for example, to outdoor settings. However, there is a strong indication, that for frequently changing environments, the pupil distance method is more robust and hence the better choice.

\section{CONCLUSION}

In this paper, we presented the first study that quantitatively compared gaze interaction with $2 \mathrm{D}$ interfaces and with $3 \mathrm{D}$ interfaces. Our findings reveal only marginal differences with regard to accuracy, usability, and mental load. With regard to user experience we observed interaction in 3D to be perceived slower, but more visually pleasing and entertaining. In addition, we compared two gaze point calculation methods to be 
applied in cases where multiple objects are displayed in the viewer's line of sight. We show that using the pupil distance to calculate the gaze point in 3D space is a robust method.

In this work, we decided to keep interaction simple and playful to obtain ecologically valid results. For future work, we plan to investigate more complex scenarios. For example, we are interested in how interaction on different depth layers and with a different number of objects affects performance.

\section{ACKNOWLEDGEMENTS}

The research leading to these results has received funding from the European Union Seventh Framework Programme ([FP7/2007-2013]) under grant agreement no. 600851.

\section{REFERENCES}

1. Alper, B., Höllerer, T., Kuchera-Morin, J., and Forbes, A. Stereoscopic highlighting: $2 \mathrm{~d}$ graph visualization on stereo displays. IEEE Trans. Vis. Comput. Graph. 17, 12 (2011), 2325-2333.

2. Ashmore, M., Duchowski, A. T., and Shoemaker, G. Efficient eye pointing with a fisheye lens. In Proc. of GI'05, Canadian Human-Computer Communications Society (2005), 203-210.

3. Bangor, A., Kortum, P. T., and Miller, J. T. An Empirical Evaluation of the System Usability Scale. International Journal on Human Computer Interaction 24, 6 (2008), 574-594.

4. Benedek, J., and Miner, T. Measuring desirability: New methods for evaluating desirability in a usability lab setting. Proc. of Usability Professionals Association 2003 (2002), 8-12.

5. Broy, N., Alt, F., Schneegass, S., Henze, N., and Schmidt, A. Perceiving layered information on $3 \mathrm{~d}$ displays using binocular disparity. In Proc. of PerDis '13, ACM (New York, NY, USA, 2013), 61-66.

6. Broy, N., André, E., and Schmidt, A. Is stereoscopic 3d a better choice for information representation in the car? In Proc. of AutoUI'12, ACM (New York, NY, USA, 2012), 93-100.

7. Duchowski, A. T., Pelfrey, B., House, D. H., and Wang, R. Measuring gaze depth with an eye tracker during stereoscopic display. In Proc. of SIGGRAPH'11, ACM (New York, NY, USA, 2011), 15-22.

8. Grossman, T., Wigdor, D., and Balakrishnan, R. Multi-finger gestural interaction with $3 \mathrm{~d}$ volumetric displays. In Proc. of UIST'04, ACM (New York, NY, USA, 2004), 61-70.

9. Häkkilä, J., Posti, M., Koskenranta, O., and Ventä-Olkkonen, L. Design and evaluation of mobile phonebook application with stereoscopic $3 \mathrm{~d}$ user interface. In CHI'13 Extended Abstracts on Human Factors in Computing Systems, ACM (2013), 1389-1394.
10. Harrison, B. L., Ishii, H., Vicente, K. J., and Buxton, W. A. S. Transparent layered user interfaces: An evaluation of a display design to enhance focused and divided attention. In Proc. of CHI'95, ACM Press / AddisonWesley (New York, NY, USA, 1995), 317-324.

11. Hart, S. G., and Staveland, L. E. Development of NASA-TLX (Task Load Index): Results of empirical and theoretical research. Human mental workload 1, 3 (1988), 139-183.

12. Hilliges, O., Kim, D., Izadi, S., Weiss, M., and Wilson, A. Holodesk: direct $3 \mathrm{~d}$ interactions with a situated see-through display. In Proc. of CHI'12, ACM (New York, NY, USA, 2012), 2421-2430.

13. Huhtala, J., Karukka, M., Salmimaa, M., and Häkkilä, J. Evaluating depth illusion as method of adding emphasis in autostereoscopic mobile displays. In Proc. of MobileHCI'11 (New York, NY, USA, 2011), 357-360.

14. Johnson, R., O’Hara, K., Sellen, A., Cousins, C., and Criminisi, A. Exploring the potential for touchless interaction in image-guided interventional radiology. In Proc. of CHI'11, ACM (New York, NY, USA, 2011).

15. Kern, D., Mahr, A., Castronovo, S., Schmidt, A., and Müller, C. Making use of drivers' glances onto the screen for explicit gaze-based interaction. In Proc. of AutoUI'10, ACM (New York, NY, USA, 2010).

16. Ki, J., and Kwon, Y.-M. 3d gaze estimation and interaction. In 3DTV Conference: The True Vision Capture, Transmission and Display of 3D Video, 2008 (2008), 373-376.

17. McIntire, J. P., Havig, P. R., and Geiselman, E. E. What is $3 \mathrm{~d}$ good for? a review of human performance on stereoscopic 3d displays. In Proceedings of Head-and Helmet-Mounted Displays XVII; and Display Technologies and Applications for Defense, Security, and Avionics VI, vol. 8383, SPIE (2012).

18. Ramasamy, C., House, D. H., Duchowski, A. T., and Daugherty, B. Using eye tracking to analyze stereoscopic filmmaking. In Adj. Proc. of SIGGRAPH'09, ACM (New York, NY, USA, 2009).

19. Reichelt, S., Häussler, R., Fütterer, G., and Leister, N. Depth cues in human visual perception and their realization in 3d displays. In SPIE Defense, Security, and Sensing, International Society for Optics and Photonics (2010), 76900B-76900B.

20. Sunnari, M., Arhippainen, L., Pakanen, M., and Hickey, S. Studying user experiences of autostereoscopic 3d menu on touch screen mobile device. In Proc. of OzCHI'12, ACM (New York, NY, USA, 2012).

21. Vidal, M., Bulling, A., and Gellersen, H. Pursuits: spontaneous interaction with displays based on smooth pursuit eye movement and moving targets. In Proc. of UbiComp'13, ACM (New York, NY, USA, 2013). 